\title{
FARADAY ROTATION AND PHOTOIONIZATION OF ZnCrSe SEMIMAGNETIC SEMICONDUCTOR*
}

\author{
W. Mac ${ }^{a}$, Nguyen The Khol ${ }^{a}, J_{\text {A. A. Gaj }}{ }^{a}$, A. Twardowski ${ }^{a}$, \\ M. DemianiuK ${ }^{b}$ and A. Stachow-UCIakowska ${ }^{a}$ \\ ${ }^{a}$ Institute of Experimental Plysics, Warsaw University \\ Hoża 69, 00-681 Warszawa, Poland \\ ${ }^{b}$ Institute of Technical Physics, WAT, Warszawa, Poland
}

The magnetoabsorption and the Faraday rotation of $\mathrm{ZnCrSe}$ were measured for $B \leq 5 \mathrm{~T}$ and $T=2 \mathrm{~K}$ in the region of photon energies lower than the fundamental absorption edge. The circular dichroism of the photoionization absorption band was observed and analyzed. The Faraday rotation was interpreted as due to the sum of contributions from interband and photoionization optical transitions."

PACS numbers: 75.50.Pp

It was reported recently [1] that a new Cr-based semimagnetic semiconductor (SMSC) ZnCrSe shows ferromagnetic $p$ - $d$ exchange between $d$-electrons and valence band electrons. This is manifested by the fact that the Zeeman splitting of free exciton has the opposite sign in comparison with "classical" SMSC (Mn, $\mathrm{Fe}$, Co-based ones).

In this paper we present the results of further magnetooptical studies on this material. We measured the magnetoabsorption and the Faraday rotation of $\mathrm{Zn}_{1-x} \mathrm{Cr}_{x}$ Se with $x=0.001$ at the temperature $T=2 \mathrm{~K}$, in the magnetic field up to $5 \mathrm{~T}$, in the region of photon energies lower than the fundamental absorption edge.

A strong absorption band related to photoionization (PI) transition was observed, in agreement with previous results of Kamińska et al. [2]. This band has its beginning at about $17000 \mathrm{~cm}^{-1}$ and extends up to about $22200 \mathrm{~cm}^{-1}$. Figure 1 shows this absorption band, which consists of two broad peaks situated at about $19000 \mathrm{~cm}^{-1}$ and $21500 \mathrm{~cm}^{-1}$. When applying a magnetic field, we see that the PI band has strong circular dichroism, that is a difference between absorption coeffcients $\alpha^{+}$and $\alpha^{-}$measured for the two circular polarizations. In the inset of Fig. 1 we plot the circular polarization degree $P=\left(I^{+}-I^{-}\right) /\left(I^{+}+I^{-}\right)$calculated for integrals $\mathrm{I}^{+}$and $\mathrm{I}^{-}$of the respective absorption coefficients over the PI structure.

"Supported by the Committee for Scientific Research (grant No. 202239101 ). 


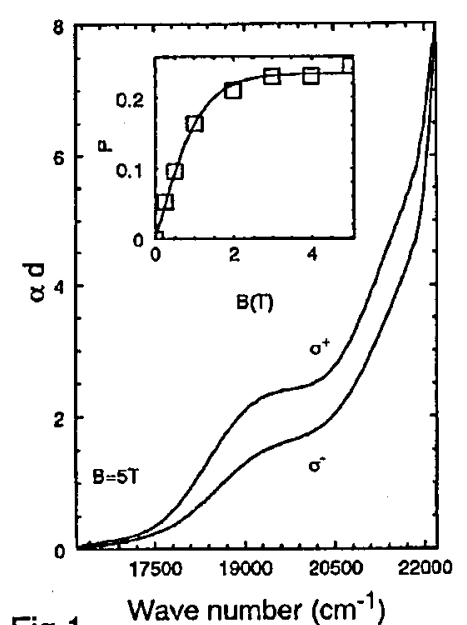

Fig. 1

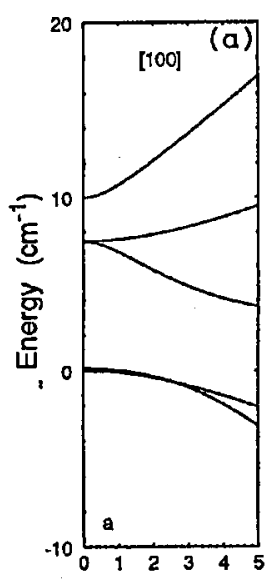

Fig. ${ }^{\mathrm{B}}(\mathrm{T})$

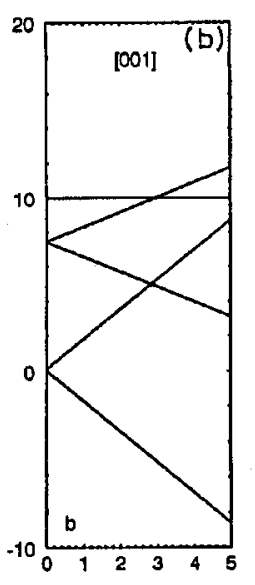

B (T)

Fig. 1. Photoionization absorption band in circular polarization of light, in the magnetic field $5 \mathrm{~T}$. In the inset: circular polarization degree of PI band vs. magnetic field $(B \|[111])$.

Fig. 2. Splitting of $\mathrm{Cr}^{++}$levels in a magnetic field. (a) $\boldsymbol{B} \|[100]$, (b) $\boldsymbol{B} \|[001]$. The Jahn-Teller distortion assumed along [001] direction.

The PI absorption band originates from the optical transition from the $\mathrm{Cr}^{++}$ configuration to the $\mathrm{Cr}^{+}$configuration. Figure 2 shows energies of the lowest lying states of a $\mathrm{Cr}^{++}$ion in $\mathrm{ZnSe}$, calculated within the crystal field theory by Twardowski et al. [3]. We see that in the first approximation, the states presented in Fig. 2 can be described as those of total momentum $j=2$ split by an axial field into a singlet $m=0$ and two doublets $m= \pm 1$ and $m= \pm 2$. This axial field (along [001] direction) is in fact a local deformation due to the Jahn-Teller effect [4], occurring in any of the three equivalent tetragonal directions. Within the approximation mentioned above, only the component of the magnetic field along the local deformation is active. Calculation performed in such a way for [111] crystallographic direction gave the dependence of circular polarization degree on magnetic field of the form of hyperbolic tangent function, which, by fitting to the experimental data, gave the $g$-factor of the $\mathrm{Cr}^{++}$state $g=2$, in agreement with the results of EPR experiments reported by Vallin and Watkins [4]. The calculation, however, gave the saturation value of the circular polarization degree within an error of $\pm 20 \%$ of its actual value. We expect that the calculation should fit better to the experimental data if we take into account not only the components of magnetic field parallel to directions $\langle 001\rangle$, but also the contribution from other components. Moreover, when calculating the circular polarization degree, we assumed that in the region of PI band, there is no contribution to the absorption coefficient from other optical transitions. Having in view that this region is very near to the fundamental absorption edge, and that the circular dichroism of the absorption edge has opposite sign, this approximation may not be very good. 
The Faraday effect was measured on sample of thickness $\approx 70 \mu \mathrm{m}$, cleaved from the same crystal, in the range of photon energies going from $11000 \mathrm{~cm}^{-1}$ up to $22500 \mathrm{~cm}^{-1}$. We could not measure at higher energies due to the strong absorption from the fundamental edge. The Faraday rotation (FR) as a function of photon energies for $B=5 \mathrm{~T}$ is plotted in Fig. 3. At lower energies, the curve is

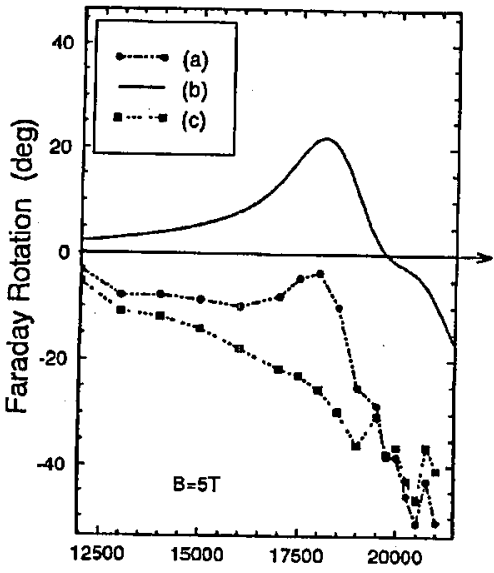

Fig.3 Wave Number $\left(\mathrm{cm}^{-1}\right)$

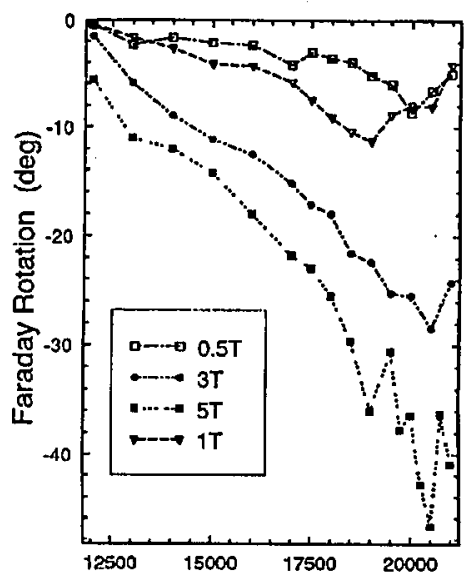

Fig.4 Wave Number $\left(\mathrm{cm}^{-1}\right)$

Fig. 3. The Faraday rotation at $B=5 \mathrm{~T}$ vs. photon energies. (a) - experiment, (b) contribution from the PI transition, (c) - contribution from the interband transition. Fig. 4. The Faraday rotation related to the interband transition for several magnetic field intensities.

rather flat. In the region of PI band, it exhibits a strong variation. We interpret the Faraday rotation, in the region concerned, as due to the sum of the contributions from the PI transition and the interband transition.

Disposing the circular dichroism of the PI band, using the Kramers-Kronig relations, we can compute the contribution from PI transition to the total FR. The results are shown in Fig. 3. By subtracting this part from the total FR, we obtain FR related to the interband optical transition as shown in Fig. 4, for some magnetic field intensities. We see that FR due to the interband transition has a negative sign*. This is in agreement with the fact that, for fundamental absorption in a magnetic field, the $\sigma^{-}$absorption edge is shifted to the lower energies, while the $\sigma^{+}$- to the higher. The sign of the Zeeman splitting of the fundamental edge in this case (in $\mathrm{ZnCrSe}$ ) is opposite to that for classical SMSC.

\section{References}

[1] W. Mac, Nguyen The Khoi, A. Twardowski, J.A. Gaj, M. Demianiuk, Acta Phys. Pol. A 82, 902 (1992).

\footnotetext{
${ }^{*}$ In our notation, $\mathrm{FR}$ of SMSC with $\mathrm{Mn}^{++}, \mathrm{Co}^{++}$, and $\mathrm{Fe}^{++}$ions is positive.
} 
[2] M. Kamińska, J. Baranowski, M. Godlewski, Inst. Phys. Conf. Ser. 43, 303 (1979). [3] A. Twardowski, T. Fries, Y. Shapira, P. Eggenkamp, H.J.M. Swagten, M. Demaniuk, J. Appl. Phys. 73, 10 (1993).

[4] J.T. Vallin, G.D.Watkins, Phys. Rev. B 9, 2051 (1974). 